\title{
CONTINUUM-WISE EXPANSIVENESS AND SPECIFICATION FOR SET-VALUED FUNCTIONS AND TOPOLOGICAL ENTROPY
}

\author{
WELINGTON CORDEIRO AND MARIA JOSÉ PACÍFICO
}

(Communicated by Nimish A. Shah)

\begin{abstract}
We define the concept of continuum-wise expansiveness for setvalued functions. We prove that for a compact metric space $X$ with positive topological dimension, if a set-valued function is $c w$-expansive, then its topological entropy is positive. We also introduce the notion of point-wise specification property for set-valued functions and prove that set-valued functions with this property have positive entropy.
\end{abstract}

\section{INTRODUCTION}

The notion of expansiveness was introduced in the middle of the twentieth century in [Utz. Expansiveness is a property shared by a large class of dynamical systems exhibiting chaotic behavior. In [Ma Mañé proved that if a compact metric space admits an expansive homeomorphism, then its topological dimension is finite. In Fa Fathi proved that if the topological dimension is greater than one, then the topological entropy of all expansive homeomorphisms is positive. In the 1990s, Kato introduced the notion of continuum-wise expansiveness for homeomorphisms [K1], and extended both results (Mañé and Fathi) to continuum-wise expansive homeomorphisms.

In $[\mathrm{BW}]$ this concept was given for flows and it was proved that some properties valid for discrete dynamics are also valid for flows. Using this definition, Keynes and Sears [KS] extended the results of Mañé for expansive flows. They proved that if a compact metric space admits an expansive flow, then its topological dimension is finite.

In $\mathrm{ACP}$ the notion of continuum-wise expansiveness for flows was introduced and the authors proved that $c w$-expansive continuous flows on compact metric spaces with topological dimension greater than one have positive entropy, extending to continuous dynamics the result of Keynes and Sears.

In view of so many interesting results relating entropy to the chaotic dynamics of single-valued functions, it is natural to ask if the concept of entropy extends to set-valued functions and, if so, if it gives nice information about the dynamics of the set-valued function.

Received by the editors October 26, 2015.

2010 Mathematics Subject Classification. Primary 37A35, 37B40, 37D45, 54C60.

Key words and phrases. Continuum-wise expansiveness, set-valued functions, entropy, specification property.

The first author was partially supported by CNPq.

The second author was partially supported by CNPq, PRONEX-Dyn.Syst., FAPERJ. 
In $\mathrm{Za}$ the author studied the asymptotic behavior of the trajectories of a setvalued dynamical system. In particular, he gave necessary and sufficient conditions for the existence of global attractors for dispersive systems as well as motivations coming from economic models.

Recently, in CMM] the authors introduced two kinds of entropy, that they call separated and spanning entropy, given by separated and spanning sets respectively. They proved that these entropies keep a number of well-known properties of the topological entropy of single-valued maps.

Also recently, in $[\mathrm{KT}$ the authors introduced the notion of entropy that resembles the classical notion of topological entropy of single-valued maps, and showed that the entropy of a set-valued function is equal to the topological entropy of the shift map on its orbit spaces. Moreover, they gave sufficient conditions for a set-valued function to have positive or infinite topological entropy.

Finally, in $[\mathrm{RT}$ the authors extended the notion of the specification property from the usual single-valued function setting to the setting of set-valued mappings and proved that specification implies topological mixing and positive entropy.

The aim of this paper is to introduce the notion of the point-wise specification property and continuum-wise expansivity for set-valued functions. We extend [K1, Theorem 5.8] to this context and give sufficient conditions for a continuum-wise expansive set-valued function to have positive entropy. We compare separated and spanning entropy defined in [CMM] with entropy defined in [KT]. We also introduce the notion of point-wise specification for set-valued functions and prove that set-valued functions with this property have positive entropy and also positive separated entropy. We point out that there are set-valued functions satisfying the specification property which second the notion given in RT but do not satisfy the point-wise specification property, as shown in Example [5.6. Moreover, there are set-valued functions satisfying the specification property which do not have positive separated entropy, as shown in Example 5.7.

This paper is organized as follows. In Section 2 we introduce the notion of continuum-wise expansive set-valued functions. In Section 3 we give sufficient conditions for a set-valued function to have positive entropy, generalizing $\mathbf{K 1}$, Theorem 5.8]. In Section 4 we exploit the notion of separated and spanning entropy and compare these kinds of entropy with the entropy of a set-valued function. Finally, in Section 5 we introduce the notion of point-wise specification for set-valued functions and prove that set-valued functions satisfying the point-wise specification property have positive entropy.

\section{Continuum-Wise expansive Set-Valued Functions}

Given a compact metric space $X$ with the metric $d$, let $2^{X}$ be the set of all non-empty subsets of $X$ with Hausdorff metric $d_{H}$, defined by

$$
d_{H}(A, B)=\max \left\{\sup _{x \in A} \inf _{y \in B} d(x, y), \sup _{y \in B} \inf _{x \in A} d(x, y)\right\},
$$

if $A, B \in 2^{X}$. Given the subsets $A$ and $B$ of $X$, recall that the distance between $A$ and $B$ is given by

$$
d(A, B)=\inf \{d(x, y) ; x \in A \text { and } y \in B\} .
$$

Let $K(X) \subset 2^{X}$ be the set of closed subsets of $X$, and $C(X) \subset K(X)$ the set of compact and connected subsets of $X$. 
We say that a function $F: X \rightarrow Y$ is a set-valued map if $Y \subset 2^{X}$. If $f: X \rightarrow Y$ is a map, we may think of $f$ as a set-valued function by defining a function $F_{f}$ : $X \rightarrow 2^{Y}$ by $F_{f}(x)=\{f(x)\}$.

For increased distinction, we will refer to a function $F: X \rightarrow 2^{Y}$ as a set-valued function and a continuous function $f: X \rightarrow Y$ as a mapping.

Let $X$ be a compact Hausdorff space and $F: X \rightarrow 2^{X}$ a set-valued map such that $F(x)$ is a compact set for each $x \in X$. Then $F$ is upper semi-continuous at $x \in X$ if for each open set $V$ containing $F(x)$ there is an open set $U$ containing $x$ such that if $y \in U$, then $F(y) \subset V$.

If $X, Y$, and $Z$ are compact metric spaces, $F: X \rightarrow 2^{Y}$ and $G: Y \rightarrow 2^{Z}$, we define $G \circ F: X \rightarrow 2^{Z}$ by

$$
G \circ F(x)=\bigcup_{y \in F(x)} G(y)
$$

Note that if $F$ and $G$ are upper semi-continuous, then $G \circ F$ is as well.

If $F: X \rightarrow 2^{X}$ satisfies $F(x)$ is a non-empty compact set for each $x \in X$, then $(X, F)$ is called a topological dynamical system. We define $F^{0}$ to be the identity on $X$, and for each $n \in \mathbb{N}, F^{n}=F \circ F^{n-1}$.

Next we recall the concept of orbit through $x \in X$ for the topological dynamical system $(X, F)$. For this, given $x \in X$, we start defining the sets

$$
\begin{gathered}
\Sigma(x)=\left\{\bar{x}=\left(\ldots, x_{-1}, x=x_{0}, x_{1}, \ldots\right) ; x_{i+1} \in F\left(x_{i}\right)\right\}, \\
\Sigma^{+}(x)=\left\{\bar{x}=\left(x=x_{0}, x_{1}, x_{2}, \ldots\right) ; x_{i+1} \in F\left(x_{i}\right)\right\}, \\
\Sigma^{-}(x)=\left\{\bar{x}=\left(x=x_{0}, x_{1}, x_{2}, \ldots\right) ; x_{i} \in F\left(x_{i+1}\right)\right\}, \\
\text { and for } n \in \mathbb{N}, \Sigma_{n}(x)=\left\{\left(x=x_{0}, \ldots, x_{n-1}\right) ; x_{i+1} \in F\left(x_{i}\right)\right\} .
\end{gathered}
$$

Definition 2.1. Given $x \in X$, the full orbit of $x$ is defined as $\operatorname{Orb}(x, F)=$ $\bigcup_{x \in X} \Sigma(x)$, the forward orbit of $x$ as $\overrightarrow{\operatorname{Orb}}(x, F)=\bigcup_{x \in X} \Sigma^{+}(x)$, and the backward orbit of $x$ as $\overleftarrow{\operatorname{Orb}}(x, F)=\bigcup_{x \in X} \Sigma^{-}(x)$. For $n \in \mathbb{N}$, the set $\operatorname{Orb}_{n}(x, F)=$ $\bigcup_{x \in X} \Sigma_{n}(x)$ is a finite orbit at $x$.

A full orbit $\operatorname{Orb}(x, F)$ is periodic if there is $n \in \mathbb{N}$ such that $x_{i}=x_{i+n}$ for all $i \in \mathbb{Z}$. If $\operatorname{Orb}(x, F)$ is periodic, the period of $x$ is the smallest number $n \in \mathbb{N}$ satisfying $x_{i}=x_{i+n}$ for all $i \in \mathbb{Z}$.

Given a set $A \subset X$ and $n \in \mathbb{N}$, we define the following orbit spaces:

$$
\begin{gathered}
\operatorname{Orb}_{n}(A, F)=\bigcup_{x \in A} \operatorname{Orb}_{n}(x, F), \quad \overrightarrow{\operatorname{Orb}}(A, F)=\bigcup_{x \in A} \overrightarrow{\operatorname{Orb}}(x, F), \\
\overleftarrow{\operatorname{Orb}}(A, F)=\bigcup_{x \in A} \overleftarrow{\operatorname{Orb}}(x, F), \quad \operatorname{Orb}(A, F)=\cup_{x \in A} \operatorname{Orb}(x, F) .
\end{gathered}
$$

We endow each of these spaces with the induced product topology.

If $\mathbb{A} \in\left\{\mathbb{Z}, \mathbb{Z}_{\geq 0}\right\}$, we consider the product space $\prod_{i \in \mathbb{A}} X$ with the metric $\rho$ defined as

$$
\rho(\bar{x}, \bar{y})=\sup _{i \in \mathbb{A}} \frac{d\left(x_{i}, y_{i}\right)}{|i|+1} .
$$

For $\prod_{i=1}^{n} X$ we define

$$
D_{n}(\bar{x}, \bar{y})=\max _{0 \leq i<n} d\left(x_{i}, y_{i}\right) .
$$

If $A \subset X$, define $F^{1}(A)=F(A)=\bigcup_{x \in A} F(x)$ and $F^{n}(A)=F \circ F^{n-1}(A)$. 
By a continuum, we mean a compact connected non-degenerate metric space.

Recall that a continuous function $f: X \rightarrow X$ is positively continuum-wise expansive if there is $\delta$ such that if $A \subset X$ is a continuous set, then there is $n \in \mathbb{N}$ with $\operatorname{diam}\left(f^{n}(A)\right)>\delta$; see $[\mathrm{K} 1$. Next we extend this concept to set-valued functions.

Definition 2.2. A topological dynamical system $(X, F)$ is positively continuumwise expansive if it satisfies:

(1) $F(x)$ is compact and connected for each $x \in X$;

(2) there is $\delta>0$ such that if $A \subset X$ is a continuum, then there is $n \in \mathbb{N}$ such that $\operatorname{diam} F^{n}(A)>\delta$.

Remark 2.3. Let $f: X \rightarrow X$ be a continuous map with $X$ a compact metric space. If we define $F_{f}: X \rightarrow 2^{X}$ by $F_{f}(x)=\{f(x)\}$, then $\left(X, F_{f}\right)$ is a topological dynamical system. By definition, a continuous map $f: X \rightarrow X$ is a positively continuum-wise expansive map if, and only if, $\left(X, F_{f}\right)$ is positively continuum-wise expansive. Moreover, since $f$ is continuous, $\left(X, F_{f}\right)$ is upper semi-continuous.

Notation. For short, we denote continuum-wise expansive by $c w$-expansive.

\section{TOPOlOGICAL ENTROPy FOR $c w$-EXPANSIVE SET-VALUED FUnCTIONS}

In this section we prove that a $c w$-expansive set-valued function defined in a metric space with positive topological dimension has positive topological entropy.

We start by recalling the notion of topological entropy for set-valued functions given in $[\mathrm{RT}$. For this, let $(X, F)$ be a topological dynamical system, $n \in \mathbb{N}$ and $\varepsilon>0$. Given $A \subset X$, we denote by $\#(A)$ the cardinality of $A$.

An $(n, \varepsilon)$-separated set for $(X, F)$ is an $(n, \varepsilon)$-separated subset of $\operatorname{Orb}_{n}(X, F)$. Define

$$
s_{n}(\varepsilon)=\sup \{\#(E) ; E \text { is an }(n, \varepsilon) \text {-separated set }\} .
$$

An $(n, \varepsilon)$-spanning set for $(X, F)$ is an $(n, \varepsilon)$-spanning subset of $\operatorname{Orb}_{n}(X, F)$. Define

$$
r_{n}(\varepsilon)=\inf \{\#(E) ; E \text { is an }(n, \varepsilon) \text {-spanning set }\} .
$$

It is proved in $[\mathbf{R T}$, Theorem 2] that

$$
\lim _{\varepsilon \rightarrow 0} \limsup _{n \rightarrow \infty} \frac{1}{n} \log r_{n}(\varepsilon)=\lim _{\varepsilon \rightarrow 0} \limsup _{n \rightarrow \infty} \frac{1}{n} \log s_{n}(\varepsilon),
$$

and the notion of entropy in [RT] is the following.

Definition 3.1. The topological entropy of $(X, F)$ is defined by

$$
h(X, F)=\lim _{\varepsilon \rightarrow 0} \limsup _{n \rightarrow \infty} \frac{1}{n} \log r_{n}(\varepsilon) .
$$

Given a continuum $A \subset X$, recall that $C(A)$ is the set of all continuum subsets of $A$.

Lemma 3.2. Let $A$ be a continuum of $X$ with $\operatorname{diam} A>c$. Then there are $A_{1}, A_{2} \in$ $C(A)$ such that $d_{H}\left(A_{1}, A_{2}\right)>\frac{c}{8}$ and

$$
\operatorname{diam}\left(A_{1}\right)=\operatorname{diam}\left(A_{2}\right)=\frac{c}{8} .
$$

Proof. Let $a_{1}, a_{2} \in A$ be points with $d\left(a_{1}, a_{2}\right)>\frac{c}{2}$. It is well known that there are $\operatorname{arcs} \alpha_{i}:[0,1] \rightarrow C(X)$ from $\left\{a_{i}\right\}$ to $A$, such that if $t<s$, then $\alpha(t) \subset \alpha(s)$, $i=1,2$. Let $t_{1}, t_{2} \in[0,1]$ be the smallest times such that diam $\alpha_{i}\left(t_{i}\right)=\frac{c}{8}, i=1,2$. Clearly, $A_{1}=\alpha\left(t_{1}\right)$ and $A_{2}=\alpha\left(A_{2}\right)$ satisfies $d_{H}\left(A_{1}, A_{2}\right)>\frac{c}{8}$. 
Next we give a technical lemma that we shall use in the proof of the main result in this section.

Lemma 3.3. Let $(X, F)$ be an upper semi-continuous topological dynamical system positively cw-expansive with constant $\eta>0$. For all $0<\delta<\eta$ and $\varepsilon>0$ there is $N \in \mathbb{N}$ such that if $A$ is a continuum and

$$
\sup _{0 \leq i \leq N} \operatorname{diam}\left(F^{i}(A)\right)<\frac{\delta}{2}
$$

then $\operatorname{diam} A<\varepsilon$.

Proof. Otherwise, there are $\varepsilon>0$ and a sequence of continuum sets $A_{n}$ such that $\operatorname{diam} A_{n} \geq \varepsilon$ and

$$
\sup _{0 \leq i \leq n} \operatorname{diam}\left(F^{i}\left(A_{n}\right)\right)<\frac{\delta}{2}, \quad \forall n \in \mathbb{N} .
$$

Since $C(X)$ is compact, we can assume that $A_{n} \rightarrow A$ with $A \subset X$ a continuum set. Here $C(X)$ is the set of compact and connected subsets of $X$. Hence $\operatorname{diam}\left(F^{n}(A)\right)<$ $\delta$ for all $n \in \mathbb{N}$. In fact, fix $n \in \mathbb{N}$ and given $x \in X$ and $\varepsilon>0$, denote by $B(x, \varepsilon)$ the usual ball with center $x$ and radius $\varepsilon$. Since $F$ is upper semi-continuous, there is $\varepsilon^{\prime}>0$ such that for all $x \in X$ if $y \in B\left(x, \varepsilon^{\prime}\right)$, then $F^{n}(y) \subset B\left(F^{n}(x), \frac{\delta}{4}\right)$. However, there is $n_{0}>n$ such that $A \subset B\left(A_{n_{0}}, \varepsilon^{\prime}\right)$, therefore $F^{n}(A) \subset B\left(F^{n}\left(A_{n_{0}}\right), \frac{\delta}{4}\right)$. Since $\operatorname{diam} F^{n}\left(A_{n_{0}}\right)<\frac{\delta}{4}$, we have $\operatorname{diam} F^{n}(A)<\delta<\eta$. This is a contradiction and proves the lemma.

If $X$ is a compact metric space, $\operatorname{dim}_{t o p}(X)$ means the topological dimension of $X$.

Theorem 3.4. Let $(X, F)$ be an upper semi-continuous cw-expansive topological dynamical system with constant of expansivity equal to $\eta>0$. If $\operatorname{dim}_{\text {top }}(X)>0$, then the topological entropy $h_{t o p}(X, F)$ is positive.

Proof. Let $A \subset C(X)$ be a non-trivial continuum set and $0<\delta<\min \{\eta$, $\operatorname{diam} A\}$. Let $N \in \mathbb{N}$ be given by Lemma 3.3 relative to $\varepsilon=\frac{\delta}{10}$. Then we can find a subcontinuum $A_{0} \subset A$ such that $\frac{\delta}{10}<\operatorname{diam}\left(A_{0}\right)<\delta$. Fix $m \in \mathbb{N}$. There is $0 \leq l \leq N$ with $\operatorname{diam}\left(F^{i_{1}}(A)\right)>\delta$. By Lemma 3.2 we can find $A_{1}, A_{2} \subset F^{l}(A)$ continuum sets with $\operatorname{diam}\left(A_{i}\right)=\frac{\delta}{8}, i \in\{1,2\}$, and $d_{h}\left(A_{1}, A_{2}\right)>\frac{\delta}{8}$. Arguing in the same way, we can find a finite collection $\left\{A_{i_{1}, i_{2}, \ldots, i_{m}} ; i_{k}=1\right.$ or 2$\}$ of continuum subsets of $X$ satisfying the following properties:

- $A_{i_{1}, \ldots, i_{j}}$ is a continuum subset of $F^{l}\left(A_{i_{1}, \ldots, i_{j-1}}\right), 1 \leq l=l\left(i_{j}-1\right) \leq N$, $i_{k}=1,2$;

- $\operatorname{diam} A_{i_{1}, \ldots, i_{j}}=\frac{\delta}{8}, i_{k}=1,2,1<j \leq m$;

- $d\left(A_{i_{1}, \ldots, i_{j}, 1}, A_{i_{1}, \ldots, i_{j}, 2}\right)>\frac{\delta}{8}, i_{k}=1,2,1<j<m$.

Then for each $\left(i_{1}, \ldots, i_{m}\right)$ we can find an orbit $\bar{a}_{i_{1}, \ldots, i_{m}}=\left(x_{0}, x_{1}, \ldots\right)$ such that for each $j \in\{1, \ldots, m\}$ it has $x_{l\left(i_{1}\right)+\cdots+l\left(i_{j}\right)} \in A_{i_{1}, \ldots, i_{j}}$. Since $1 \leq l(k) \leq N$ for each $k=1, \ldots, m$, we have that the set

$$
E_{m}=\left\{\bar{a}_{i_{1}, \ldots, i_{m}} ; i_{j}=1 \text { or } 2\right\}
$$


is a $\left(\frac{\delta}{8}, N m\right)$-separated set with $2^{m}$ elements. Thus $s_{N m}\left(\frac{\delta}{8}\right) \geq 2^{m}$ and

$$
\begin{aligned}
\limsup _{n \rightarrow \infty} \frac{1}{n} \log s_{n}\left(\frac{\delta}{8}\right) & \geq \limsup _{m \rightarrow \infty} \frac{1}{N m} \log s_{N m}\left(\frac{\delta}{8}\right) \\
& \geq \limsup _{m \rightarrow \infty} \frac{1}{N m} \log s_{N m}\left(\frac{\delta}{8}\right) \\
& \geq \limsup _{m \rightarrow \infty} \frac{1}{N m} \log 2^{m}=\frac{1}{N} \log 2>0 .
\end{aligned}
$$

Hence $h(X, F)>0$. The proof of Theorem 3.4 is complete.

Combining Theorem 3.4 with [RT, Theorems 3.1 and 3.5] we obtain the following generalization to the setting of set-valued functions of [K1, Theorem 5.8].

Corollary 3.5. Let $(X, F)$ be an upper semi-continuous topological dynamical system cw-expansive with $\operatorname{dim}_{\text {top }}(X)>0$. Then

(1) If $\vec{\sigma}: \overrightarrow{\mathrm{Orb}}(X, F) \rightarrow \overrightarrow{\mathrm{Orb}}(X, F)$ is the shift map defined as

$$
\vec{\sigma}\left(x_{0}, x_{1}, \ldots\right)=\left(x_{1}, x_{2}, \ldots\right),
$$

then $h(\vec{\sigma})>0$.

(2) If $\overleftarrow{\sigma}: \overleftarrow{\mathrm{Orb}}(X, F) \rightarrow \overleftarrow{\mathrm{Orb}}(X, F)$ is the shift map defined as

$$
\overleftarrow{\sigma}\left(\ldots, x_{-1}, x_{0}\right)=\left(\ldots, x_{-2}, x_{-1}\right)
$$

then $h(\overleftarrow{\sigma})>0$

(3) If $\sigma: \operatorname{Orb}(X, F) \rightarrow \operatorname{Orb}(X, F)$ is the shift map defined as

$$
\sigma\left(\left\{x_{i}\right\}_{i=-\infty}^{\infty}\right)=\left(\left\{y_{i}\right\}_{i=-\infty}^{\infty}\right) \text {, }
$$

where $y_{i}=x_{i+1}$, then $h(\sigma)>0$.

Example 3.6. Let $f: S^{1} \rightarrow S^{1}$ be a rotation of the circle. Define $F: S^{1} \rightarrow 2^{S^{1}}$ by $F(t)=[t, f(t)]$. Then $\left(S^{1}, F\right)$ is $c w$-expansive, but $\vec{\sigma}$ and $\sigma$ are not $c w$-expansive maps. Indeed, there is $n \in \mathbb{N}$ such that for all points $t \in S^{1}$ we have $F(t)=$ $S^{1}$. Therefore for each continuum $A \subset S^{1}$ we obtain that $F^{n}(A)=S^{1}$ implying that $(X, F)$ is $c w$-expansive. On the other hand, for each $t \in S^{1}$ we have that $I^{ \pm}(t)=(t, t, t, t, \ldots) \in \Sigma^{+}(t) \cap \Sigma^{-}(t)$ and $I(t)=(\ldots, t, t, t, t, \ldots) \in \Sigma(t)$. Given $\delta>0$, choose $t<t^{\prime}$ such that $\operatorname{diam}\left[t_{1}, t_{2}\right]<\delta$. Then $B=\left\{I(t) ; t \in\left[t_{1}, t_{2}\right]\right\}$ and $B^{ \pm}=\left\{I^{ \pm}(t) ; t \in\left[t_{1}, t_{2}\right]\right\}$ are continuum subsets of $\overrightarrow{\mathrm{Orb}}(X, F)$.

But $\vec{\sigma}^{n}\left(B^{ \pm}\right)=\overleftarrow{\sigma}^{n}\left(B^{ \pm}\right)=B^{ \pm}$and $\sigma^{n}(B)=B$ for all $n \in \mathbb{N}$. Hence $\vec{\sigma}, \overleftarrow{\sigma}$ and $\sigma$ are not positively $c w$-expansives maps.

If $A \subset X$ is a continuum, then $\pi(A)=\bigcup_{x \in A} \Sigma^{+}(x)$ is a subcontinuum of $\overrightarrow{\operatorname{Orb}}(X, F)$. However, if $A$ is a subcontinuum of $\overrightarrow{\operatorname{Orb}}(X, F)$, then $\pi^{-1}(A)$ may not be a continuum in $X$. In fact, it can be only one point: in Example 5.6 described below, for each $t \in S^{1}, \Sigma^{+}(t)$ is a continuum but $\pi^{-1}\left(\Sigma^{+}(t)\right)=\{t\}$.

Proposition 3.7. Let $(X, F)$ be an upper semi-continuous topological dynamical system and let $\vec{\sigma}: \overrightarrow{\operatorname{Orb}}(X, F) \rightarrow \overrightarrow{\operatorname{Orb}}(X, F), \sigma: \operatorname{Orb}(X, F) \rightarrow \operatorname{Orb}(X, F)$ be the shift maps defined above. Then

(1) If $\vec{\sigma}$ is a positively cw-expansive map, then $(X, F)$ is a positively cwexpansive topological dynamical system.

(2) If $\sigma$ is a positively cw-expansive map, then $(X, F)$ is a positively cwexpansive topological dynamical system. 
Proof. (1) Let $\delta>0$ be a cw-expansive constant to $\vec{\sigma}$. If $A \subset X$ is a continuum set, then $\pi(A)=\bigcup_{x \in A} \Sigma^{+}(x)$ is a subcontinuum of $\overrightarrow{\mathrm{Orb}}(X, F)$. By the choice of $\delta$, there is $n_{1} \in \mathbb{N}$ such that $\operatorname{diam}\left(\vec{\sigma}^{n_{1}}(\pi(A))\right)>\delta$. Since $\pi(A)$ is a compact set, we can find $\bar{x}, \bar{y} \in \pi(A)$ such that $\rho\left(\vec{\sigma}^{n_{1}}(\bar{x}), \vec{\sigma}^{n_{1}}(\bar{y})\right)>\delta$. By the definition of $\rho$ in (11), there is $n_{2} \in \mathbb{N}$ such that

$$
\frac{d\left(x_{n_{1}+n_{2}}, y_{n_{1}+n_{2}}\right)}{n_{2}+1}>\delta .
$$

Since $x_{n_{1}+n_{2}}, y_{n_{1}+n_{2}} \in F^{n_{1}+n_{2}}(A)$, we get $\operatorname{diam}\left(F^{n_{1}+n_{2}}(A)\right)>\left(n_{2}+1\right) \delta \geq \delta$, proving (1).

(2) The proof is similar to the previous item and we leave it to the reader.

\section{SeParated And SPANning topological Entropy}

In this section we exploit the notion of separated and spanning entropy introduced in CMM]. For this, let $X$ be a compact metric space and let $F: X \rightarrow 2^{X}$ be a set-valued function defined on $X$. Given $n \in \mathbb{N}$, we consider the product space $\prod_{i=1}^{n} X$ endowed with the metric $D_{n}$ defined in (2).

For $n \in \mathbb{N}$ define the map $d_{n}: X \times X \rightarrow \mathbb{R}$ by

$$
d_{n}(x, y)=\inf \left\{D_{n}(\bar{x}, \bar{y}) ; \bar{x} \in \Sigma_{n}(x) \text { and } \bar{y} \in \Sigma_{n}(y)\right\} .
$$

These maps are metrics in the single-valued case but in general they are only semimetrics; see [Do, Gi] and CMM, Example 4.5].

The $\varepsilon$-ball centered at $x \in X$ and radius $\varepsilon$ with respect to $d_{n}$ is given by

$$
B_{n}[x, \varepsilon]=\left\{y \in X ; d_{n}(x, y) \leq \varepsilon\right\} .
$$

For $\varepsilon>0$ and $n \in \mathbb{N}$ we say that $E \subset X$ is an $\left(d_{n}, \varepsilon\right)$-separated set if

$$
B_{n}[x, \varepsilon] \cap E=\{x\}, \quad \forall x \in E .
$$

We let $S_{n}(\varepsilon)$ be the largest cardinality of a $\left(d_{n}, \varepsilon\right)$-separated set and define the $s e$-separated entropy of $(X, F)$ by

$$
h_{s e}(X, F)=\lim _{\varepsilon \rightarrow 0} \limsup _{n \rightarrow \infty} \frac{1}{n} \log S_{n}(\varepsilon) .
$$

For $\varepsilon>0$ and $n \in \mathbb{N}$ we say that $G \subset X$ is a $\left(d_{n}, \varepsilon\right)$-spanning set if

$$
X=\bigcup_{x \in G} B_{n}[x, \varepsilon] .
$$

We let $R_{n}(\varepsilon)$ be the smallest cardinality of a $\left(d_{n}, \varepsilon\right)$-spanning set and define the spanning entropy of $(X, F)$ by

$$
h_{s p}(X, F)=\lim _{\varepsilon \rightarrow 0} \limsup _{n \rightarrow \infty} \frac{1}{n} \log R_{n}(\varepsilon) .
$$

It is proved in CMM, Theorem 3.5] that $h_{s p}(X, F) \leq h_{s e}(X, F)$ and that they coincide if and only if $d_{n}$ is a metric [CMM, Theorem 3.6]. Next, we compare the separated entropy to the entropy of a topological dynamical system $(X, F)$ given in Definition 3.1

Theorem 4.1. Let $(X, F)$ be a topological dynamical system. Then $h_{\text {se }}(X, F) \leq$ $h(X, F)$. 
Proof. Let $n \in \mathbb{N}$ and $\varepsilon>0$ be given. Let $E$ be a $\left(d_{n}, \varepsilon\right)$-separated set with maximal cardinality. For each $x \in E$ choose $\bar{x} \in \Sigma(x)$. Let $G \subset \operatorname{Orb}(E, F)$ be the set of all these sequences and

$$
H=\{\overline{x(n)} ; \bar{x} \in G\} .
$$

If $\overline{x(n)}, \overline{y(n)} \in H$, since $x \in E$ we get $B_{n}[x, \varepsilon] \cap E=\{x\}$, and since also $y \in E$ we get

$$
\inf \left\{d_{n}(a, b) ; a \in \Sigma_{n}(x) \text { and } b \in \Sigma_{n}(y)\right\}>\varepsilon \text {. }
$$

Since $\overline{x(n)} \in \Sigma_{n}(x)$ and $\overline{y(n)} \in \Sigma_{n}(y)$, we get $d_{n}(x(n), y(n))>\varepsilon$. Hence $H$ is an $\varepsilon$-separated subset of $\operatorname{Orb}_{n}(X, F)$.

Thus, since we proved that $S_{n}(\varepsilon) \leq s_{n}(\varepsilon)$ for all $n \in \mathbb{N}$ and $\varepsilon>0$, we conclude that $h_{s e}(X, F) \leq h(X, F)$.

Example 4.2. Here we give an example of a dynamical system $(X, F)$ such that $h_{s e}<h(X, F)$. Let $f: S^{1} \rightarrow S^{1}$ be defined by $f(t)=t^{2}$. Define a topological dynamical system $\left(S^{1}, F\right)$ by $F(t)=B[f(t), \delta]$ for some $\delta>0$. So there is $n \in \mathbb{N}$ (depending only on $\delta$ ) such that $F^{n}(t)=S^{1}$ for all $t \in S^{1}$. Therefore, $\left(S^{1}, F\right)$ is a $c w$-expansive dynamical system and since $\operatorname{dim} S^{1}=1$, Theorem 3.4 implies that $h\left(S^{1}, F\right)>0$. On the other hand, [CMM, Example 4.9] implies that $h_{s e}=0$.

\section{Point-Wise SPECificAtion PROPERTy FOR SET-VALUEd FUnCtions}

In this section we introduce the notion of the point-wise specification property for set-valued functions and prove that set-valued functions satisfying this property have positive entropy. For this, we start by giving the definition of specification introduced in $[\mathrm{RT}$.

Definition 5.1. A topological dynamical system $(X, F)$ has the (periodic) specification property if for each $\varepsilon>0$ there is $M>0$ such that, for any $x^{1}, \ldots, x^{n} \in X$, any $a_{1} \leq b_{1}<\cdots<a_{n} \leq b_{n}$ with $a_{i+1}-b_{i}>M$, and any orbits $\left(x_{j}^{i}\right)_{j=0}^{\infty}$ (and for any $\left.P>M+b_{n}-a_{1}\right)$, there exists a point $z \in X$ that has an orbit $\left(z_{j}\right)_{j=0}^{\infty}$ such that $d\left(z_{j}, x_{j}^{i}\right)<\varepsilon$, for $i \in\{1, \ldots, n\}$ and $a_{i} \leq j \leq b_{i}$ (and $z_{P}=z$ ).

Remark 5.2. The notion of the periodic specification property was called the specification property in $[\mathrm{RT}$. Note that the periodic specification property implies the specification property.

The following result was proved for the periodic specification property in $[\mathrm{RT}]$. The proof for the specification property is essentially the same, and we leave it to the reader.

Theorem $5.3([\mathrm{RT}$, Theorem 5$])$. Let $(X, F)$ be a topological dynamical system such that $\#(X) \geq 2$. If $(X, F)$ has the specification property, then $h(X, F)>0$.

Next we define the point-wise specification property for set-valued functions.

Definition 5.4. A topological dynamical system $(X, F)$ has the point-wise specification property if for each $\varepsilon>0$ there is $M>0$ such that, for any $x^{1}, \ldots, x^{n} \in X$ and any $a_{1}<a_{2}<\cdots<a_{n}$ with $a_{i+1}-a_{i}>M$, there exists a point $z \in X$ such that $d_{H}\left(F^{j}(z),\left\{x^{i}\right\}\right)<\varepsilon$, for $i \in\{1, \ldots, n\}$.

Remark 5.5. Let $f: X \rightarrow X$ be a continuous function on a compact metric space $X$. If $f$ has the specification property, then $\left(X, F_{f}\right)$ has the pointwise specification property. Here $F_{f}: X \rightarrow 2^{X}$ is the set-valued function induced by $f$, defined by $F_{f}(x)=\{f(x)\}$. 
Example 5.6. We will show a simple topological dynamical system with the periodic specification property which does not have the pointwise specification property. Let $X$ be a compact and connected metric space with $\operatorname{diam}(X)>1$ and let $F: X \rightarrow 2^{X}$ be defined by $F(x)=X$, for each $x \in X$. On the one hand, let $\varepsilon>0$ be given and take $M=0, x^{1}, \ldots, x^{n} \in X, a_{1} \leq b_{1}<\cdots<a_{n} \leq b_{n}$ with $a_{i+1}-b_{i}>0$, orbits $\left(x_{j}^{i}\right)_{j=0}^{\infty}$, and $P>1+b_{n}-a_{1}$. If we choose $z=x_{a_{1}}^{1}$, since $F(x)=X$ we can find an orbit $\left(z_{j}\right)_{j=0}^{\infty}$ of $z$ such that $z_{j}=x_{j}^{i}$, for $i \in\{1, \ldots, n\}$, $a_{i} \leq j \leq b_{i}$, and $z_{P}=z$. On the other hand, assume that $(X, F)$ has the strong specification property. Take $M \geq 0$ for $\varepsilon=\frac{1}{2}$. Choose $x \in X$ and take $a_{1}=1$. For any $z \in X$, since $F(z)=X$ we have

$$
d_{H}\left(F^{a_{1}}(z),\{x\}\right)=d_{H}(X,\{x\})>\frac{1}{2}=\varepsilon,
$$

leading to a contradiction. Therefore, $(X, F)$ does not have the point-wise specification property.

Example 5.7. The periodic specification property does not imply $h_{s e}(X, F)>0$. Indeed, applying [RT, Theorem 5] to the previous example, we get $h(X, F)>0$. Since $F^{n}(x)=X$ for all $n \geq 1, d_{n}(x, y)=d(x, y)$. Hence $S_{n}(\varepsilon)=C_{\varepsilon}$, for all $n \in \mathbb{N}$. Therefore,

$$
\limsup _{n \rightarrow \infty} \frac{1}{n} \log S_{n}(\varepsilon)=\limsup _{n \rightarrow \infty} \frac{1}{n} \log C_{\varepsilon}=0 \Rightarrow h_{s e}(X, F)=0 .
$$

Definition 5.8. A topological dynamical system $(X, F)$ is topologically mixing if, for any non-empty subsets $U$ and $V$ in $X$, there is $M \in \mathbb{N}$ such that for each $m>M$ there is $x_{0} \in U$ with orbit $\left(x_{j}\right)_{j=0}^{\infty}$ such that $x_{m} \in V$; see [RT].

Theorem 5.9. Let $(X, F)$ be a topological dynamical system. If $(X, F)$ has the point-wise specification property, then $(X, F)$ is topologically mixing.

Proof. Let $U$ and $V$ be open sets in $X$ and take $x \in U, y \in V$ and $\varepsilon>0$ such that $B(x, \varepsilon) \subset U$ and $B(y, \varepsilon) \subset V$. Let $M$ be given by the point-wise specification property. By the point-wise specification property, for each $m \in \mathbb{N}$ there is $z^{m} \in X$ such that $d_{H}\left(\left\{z^{m}\right\},\{x\}\right)<\varepsilon$ and $d_{H}\left(F^{M+m}\left(z^{m}\right),\{y\}\right)<\varepsilon$. Take $\left(z_{n}^{m}\right)_{n=0}^{\infty} \in$ $\Sigma\left(z^{m}\right)$. Since $z_{0}^{m}=z^{m}$ and $z_{M+m}^{m} \in F^{M+m}\left(z^{m}\right)$,

$$
d\left(z_{0}^{m}, x\right)=d\left(z^{m}, x\right)=d_{H}\left(\left\{z^{m}\right\},\{x\}\right)<\varepsilon
$$

and

$$
d\left(z_{M+m}^{m}, y\right) \leq d_{H}\left(F^{M+m}\left(z^{m}\right),\{y\}\right)<\varepsilon .
$$

Hence $z_{0}^{m} \in U$ and $z_{M+m}^{m} \in V$. Then $(X, F)$ is topologically mixing.

Theorem 5.10. Let $(X, F)$ be a topological dynamical system with cardinality of $X$ greater than 2. If $(X, F)$ has the point-wise specification property, then $h_{\text {se }}(X, F)$ is positive.

Proof. Take $x \neq y \in X$ and $0<\varepsilon<\frac{d(x, y)}{3}$, and let $M$ be given by the point-wise specification property for this $\varepsilon$. For $n \in \mathbb{N}$, define

$$
A_{n}=\left\{\left(c^{1}, \ldots, c^{n}\right) ; c^{i}=x, y\right\} .
$$

Then $A_{n}$ has $2^{n}$ elements. For each $c=\left(c^{1}, \ldots, c^{n}\right) \in A_{n}$, if $a_{i}=(i-1) M$, there is a point $z^{c} \in X$ such that

$$
d_{H}\left(F^{(i-1) M}\left(z^{c}\right),\left\{c^{i}\right\}\right)<\varepsilon .
$$


Hence if $c \neq d \in A_{n}$, there is $i_{0} \in\{1, \ldots, n\}$ such that $c^{i_{0}} \neq d^{i_{0}}$. Therefore, if $\overline{z_{c}} \in \Sigma\left(z^{c}\right)$ and $\overline{z^{d}} \in \Sigma\left(z^{d}\right)$,

$$
D_{n M}\left(\bar{z}^{c}, \bar{z}^{d}\right)=\sup _{0 \leq i \leq(n-1) M} d\left(z_{i}^{c}, z_{i}^{d}\right) \geq d\left(z_{\left(i_{0}-1\right) M}^{c}, z_{\left(i_{0}-1\right) M}^{d}\right) .
$$

Since $z_{\left(i_{0}-1\right) M}^{c} \in F^{\left(i_{0}-1\right) M}\left(z^{c}\right)$ and $z_{\left(i_{0}-1\right) M}^{d} \in F^{\left(i_{0}-1\right) M}\left(z^{d}\right)$,

$$
d\left(z_{\left(i_{0}-1\right) M}^{c}, c^{i_{0}}\right) \leq d_{H}\left(F^{\left(i_{0}-1\right) M}\left(z^{c}\right),\left\{c^{i_{0}}\right\}\right)<\varepsilon
$$

and

Thus

$$
d\left(z_{\left(i_{0}-1\right) M}^{d}, d^{i_{0}}\right) \leq d_{H}\left(F^{\left(i_{0}-1\right) M}\left(z^{d}\right),\left\{d^{i_{0}}\right\}\right)<\varepsilon
$$

$d\left(z_{\left(i_{0}-1\right) M}^{c}, z_{\left(i_{0}-1\right) M}^{d}\right) \geq d\left(c^{i_{0}}, d^{i_{0}}\right)-d\left(z_{\left(i_{0}-1\right) M}^{c}, c^{i_{0}}\right)-d\left(z_{\left(i_{0}-1\right) M}^{d}, d^{i_{0}}\right)>3 \varepsilon-\varepsilon-\varepsilon=\varepsilon$.

By (3), $D_{n M}\left(\bar{z}^{c}, \bar{z}^{d}\right)>\varepsilon$. Hence if

$$
E_{n}=\left\{z_{c} ; c \in A_{n}\right\},
$$

we have that cardinality of $E_{n}$ is $2^{n}$ and for each $z, w \in E_{n}$ we have $d_{n}(z, w)>\varepsilon$, i.e., $B_{n}[z, \varepsilon] \cap E_{n}=\{z\}$ for each $z \in E_{n}$. Therefore, $E_{n}$ is a $\left(d_{n}, \varepsilon\right)$-separated set. Hence for a fixed $\varepsilon>0$ we get

$$
\begin{aligned}
\limsup _{n \rightarrow \infty} \frac{1}{n} \log \left(S_{n}(\varepsilon)\right) & \geq \limsup _{n \rightarrow \infty} \frac{1}{n M} \log \left(S_{n M}(\varepsilon)\right) \\
& \geq \limsup _{n \rightarrow \infty} \frac{1}{n M} \log \left(2^{n}\right) \\
& =\limsup _{n \rightarrow \infty} \frac{1}{M} \log (2)=\frac{1}{M} \log (2) .
\end{aligned}
$$

Then $h_{s e}(X, F)$ is positive and we conclude the proof.

Combining Theorems 5.10 and 4.1 we have

Corollary 5.11. Let $(X, F)$ be a topological dynamical system with cardinality of $X$ greater than 2. If $(X, F)$ has the point-wise specification property, then $h(X, F)$ is positive.

Combining Corollary 5.11 and [KT, Theorem 3.1, Theorem 3.5] we have

Corollary 5.12. Let $(X, F)$ be a topological dynamical system with cardinality of $X$ greater than 2. If $(X, F)$ has the point-wise specification property, then $h(\overleftarrow{\sigma})>0$ $h(\vec{\sigma})>0$, and $h(\sigma)>0$.

Example 5.13 (A non-trivial topological dynamical system with the point-wise specification property). Let $f: \mathbb{T}^{2} \rightarrow \mathbb{T}^{2}$ be the classical Anosov linear on the torus. Define $F: \mathbb{T}^{2} \rightarrow 2^{\mathbb{T}^{2}}$ by $F(x)=\{f(x)\}$ if $x$ is a periodic point for $f$, and by $F(x)=\mathbb{T}^{2}$ if $x$ is not a periodic point for $f$. Since $f$ is an Anosov transitive homeomorphism, there is the periodic specification property. Let $\varepsilon>0$ be given and let $M>0$ be given by the periodic specification property for $f$. Let $x^{1}, \ldots, x^{n} \in \mathbb{T}^{2}$ and $a_{1}<a_{2}<\cdots<a_{n}$ with $a_{i+1}-a_{i}>M$ be given. For each $1 \leq i \leq n$ take $y^{i}=f^{-a_{i}}\left(x^{i}\right)$. By the choice of $M$, there is $z \in \mathbb{T}^{2}$, a periodic point of $f$, such that $d\left(f^{a_{i}}(z), f^{a_{i}}\left(y^{i}\right)\right)<\varepsilon$, for all $1 \leq i \leq n$. Hence for each $1 \leq i \leq n$,

$$
d_{H}\left(F^{a_{i}}(z),\left\{x^{i}\right\}\right)=d_{H}\left(\left\{f^{a_{i}}(z)\right\},\left\{f^{a_{i}}\left(y^{i}\right)\right\}\right)=d\left(f^{a_{i}}(z), f^{a_{i}}\left(y^{i}\right)\right)<\varepsilon .
$$

Therefore $(X, F)$ has the point-wise specification property. 


\section{ACKNOWLEDGMENTS}

This work was developed while the first author held a postdoctoral position at Pennsylvania State University. He is grateful for the CNPq grant and thanks the Pennsylvania State University math department for its hospitality during this period.

\section{RefERENCES}

[ACP] A. Arbieto, W. Cordeiro, and M. J. Pacifico, cw-expansivity and entropy for flows, arXiv:1507.01198.

[AKM] R. L. Adler, A. G. Konheim, and M. H. McAndrew, Topological entropy, Trans. Amer. Math. Soc. 114 (1965), 309-319. MR0175106

[B1] Rufus Bowen, Entropy for group endomorphisms and homogeneous spaces, Trans. Amer. Math. Soc. 153 (1971), 401-414. MR0274707

[B2] Rufus Bowen, Periodic orbits for hyperbolic flows, Amer. J. Math. 94 (1972), 1-30. MR0298700

[BW] Rufus Bowen and Peter Walters, Expansive one-parameter flows, J. Differential Equations 12 (1972), 180-193. MR0341451

[CMM] Dante Carrasco-Olivera, Roger Metzger Alvan, and Carlos Arnoldo Morales Rojas, Topological entropy for set-valued maps, Discrete Contin. Dyn. Syst. Ser. B 20 (2015), no. 10, 3461-3474, DOI 10.3934/dcdsb.2015.20.3461. MR3411534

[Fa] Albert Fathi, Expansiveness, hyperbolicity and Hausdorff dimension, Comm. Math. Phys. 126 (1989), no. 2, 249-262. MR.1027497

[CDY] Welington Cordeiro, Manfred Denker, and Michiko Yuri, A note on specification for iterated function systems, Discrete Contin. Dyn. Syst. Ser. B 20 (2015), no. 10, 3475-3485, DOI 10.3934/dcdsb.2015.20.3475. MR.3411535

[Do] Tomasz Downarowicz, Entropy in dynamical systems, New Mathematical Monographs, vol. 18, Cambridge University Press, Cambridge, 2011. MR2809170

[Gi] Basil E. Gillam, A new set of postulates for euclidean geometry, Revista Ci., Lima 42 (1940), 869-899. MR0004473

[K1] Hisao Kato, Continuum-wise expansive homeomorphisms, Canad. J. Math. 45 (1993), no. 3, 576-598, DOI 10.4153/CJM-1993-030-4. MR.1222517

[KT] J. M. Kelly and T. Tennant, Topological entropy of set-valued functions, arXiv: $1509.08413 \mathrm{v} 1$ (2015).

[KS] H. B. Keynes and M. Sears, Real-expansive flows and topological dimension, Ergodic Theory Dynamical Systems 1 (1981), no. 2, 179-195. MR661818

[Ma] Ricardo Mañé, Expansive homeomorphisms and topological dimension, Trans. Amer. Math. Soc. 252 (1979), 313-319, DOI 10.2307/1998091. MR534124

[RT] B. E. Raines and T. Tennant, The specification property on a set-valued map an its inverse limit, arXiv: 1509.08415v1 (2015).

[Re] William L. Reddy, Pointwise expansion homeomorphisms, J. London Math. Soc. (2) 2 (1970), 232-236. MR0258009

[Sc] S. Schwartzman, On transformations groups, Dissertation, Yale University, 1952.

[Utz] W. R. Utz, Unstable homeomorphisms, Proc. Amer. Math. Soc. 1 (1950), 769-774. MR 0038022

[Za] Alexander J. Zaslavski, Convergence of trajectories of discrete dispersive dynamical systems, Commun. Math. Anal. 4 (2008), no. 1, 10-19. MR2365919

Department of Mathematics, The Pennsylvania State University, State College, PENNSYlVANia 16802

E-mail address: wud11@psu.edu

Instituto de Matemática, Universidade Federal do Rio de Janeiro, C. P. 68.530 CEP

21.945-970, Rio DE JANEIRo, RJ, BRAZIL

E-mail address: pacifico@im.ufrj.br 